\title{
ASSOCIAÇÕES HERBICIDAS APLICADAS NA CULTURA DO MILHO PIPOCA EM DIFERENTES ESTÁDIOS DE DESENVOLVIMENTO
}

\author{
THIAGO MARÇAL MAIA ${ }^{1}$, GUILHERME BRAGA PEREIRA BRAZ ${ }^{1}$, \\ FELLIPE GOULART MACHADO ${ }^{2}$, ALESSANDRO GUERRA DA SILVA ${ }^{1}$, \\ CHRISTIANO LIMA LOBO DE ANDRADE ${ }^{1}$ e GUSTAVO ANDRÉ SIMON ${ }^{1}$
}

\author{
'Universidade de Rio Verde, Rio Verde, GO,Brasil, agromaia@hotmail.com, \\ guilhermebrag@gmail.com,silvaag@yahoo.com.br,christiano.instrutoria@gmail.com,simon@unirv.edu.br \\ ${ }^{2}$ Universidade Estadual de Maringá, Maringá, PR, Brasil, fellipe.goulart@outlook.com
}

Revista Brasileira de Milho e Sorgo, v.18, n.3, p. 350-363, 2019

\begin{abstract}
RESUMO - Para assegurar a produtividade e qualidade do milho pipoca é fundamental evitar a interferência das plantas daninhas. Para isto, é necessário utilizar herbicidas que, além de proporcionarem bom controle, sejam seletivos à cultura. O objetivo do trabalho foi avaliar a seletividade de associações herbicidas para a cultura do milho pipoca em diferentes estádios. Foram conduzidos dois experimentos a campo no delineamento de blocos casualizados, com quatro repetições. O Experimento I foi instalado em arranjo fatorial $7 \times 2$, sendo que o primeiro fator correspondeu a aplicação de mesotrione, tembotrione e nicosulfuron em duas doses de cada herbicida. O segundo fator foi constituído pela associação ou não com atrazine $\left(2000 \mathrm{~g} \mathrm{ha}^{-1}\right)$. O Experimento II foi instalado em arranjo fatorial 4 x 3+1, no qual o primeiro fator foi constituído da aplicação dos herbicidas mesotrione + atrazine, tembotrione + atrazine, nicosulfuron + atrazine e atrazine. $\mathrm{O}$ segundo fator foi composto pelas aplicações em pós-emergência em três estádios fenológicos do milho pipoca (V2, V4 e V6). O tratamento adicional consistiu da testemunha sem aplicação de herbicidas. Pode se constatar que herbicidas com espectro sobre gramíneas aplicados em associação com atrazine proporcionaram maiores injúrias às plantas de milho pipoca. Além disso, estes herbicidas, quando aplicados nas maiores doses, causaram redução nos valores de massa seca de parte aérea das plantas de milho pipoca. A produtividade do híbrido VYP $212^{\circledR}$ não foi afetada pela aplicação dos tratamentos, demonstrando que as associações herbicidas testadas podem ser utilizadas no manejo de plantas daninhas em diferentes estádios de aplicação. Palavras-chave: controle químico, milhos especiais, seletividade, Zea mays.
\end{abstract}

\section{HERBICIDES ASSOCIATIONS APPLIED IN POPCORN CROP IN DIFFERENT DEVELOPMENT STAGES}

\begin{abstract}
To ensure the productivity and quality of popcorn corn, it is essential avoid weed interference, for this it is necessary use herbicides that show good control in weed and being selective for the crop. The objective of this work was to evaluate the selectivity of herbicides associations for popcorn corn in different development stages. Two field experiments were conducted in a randomized block design with four replications. The experiment I was installed in a 7 x 2 factorial arrangement, the first factor corresponded to the application of mesotrione, tembotrione and, nicosulfuron in two doses of each herbicide. The second factor was constituted by the association or not with atrazine $\left(2000 \mathrm{~g} \mathrm{ha}^{-1}\right)$. The experiment II was installed in a $4 \times 3+1$ factorial arrangement, where the first factor was the application of the herbicides mesotrione + atrazine, tembotrione + atrazine, nicosulfuron + atrazine and atrazine. The second factor was composed by postemergence applications in three phenological stages of popcorn (V2, V4 and V6). The additional treatment consisted of the control without herbicide application. In the results the herbicides with grasses spectrum applied in association with atrazine provided injuries in the popcorn corn plants. Moreover, these herbicides, when applied at the higher dosages, reduce the aerial dry mass values of the popcorn corn plants. The VYP $212^{\circledR}$ hybrid yield was not affected by the application of the treatments, showing that the herbicides associations can be used in the management of weeds of the popcorn corn in different stages of application.
\end{abstract}

Keywords: chemical control, special corn, selectivity, Zea mays. 
O milho pipoca (Zea mays L.) pertence à família Poaceae e é oriundo da mesma espécie botânica do milho comum (Silveira et al., 2015). Este cereal, além do consumo como pipoca, também pode ser utilizado como fonte na fabricação de alimentos básicos como fubás, canjicas, farinhas e óleos. Além disso, o seu xarope de glucose é utilizado na produção de balas, gomas de mascar, entre outros (Sologuren, 2015).

A planta de milho pipoca, quando comparada à do milho comum, se caracteriza por apresentar menor vigor, grãos menores, maior susceptibilidade a pragas e doenças e a produção de espigas de tamanho menor, além da maior capacidade de expansão dos grãos (Iken \& Amusa, 2010). O milho pipoca apresenta boa adaptabilidade às diversas condições de ambiente de produção em que é submetido, sendo por vezes as recomendações de cultivo oriundas do milho comum (Freitas et al., 2009).

Para o manejo de plantas daninhas, geralmente há informações disponíveis nas bulas dos herbicidas para aplicação nas culturas que apresentam seletividade. Nas bulas dos principais herbicidas comerciais disponíveis para uso em milho comum, não há especificações sobre o uso em milho pipoca. Exempos como atrazine $\left(\right.$ Gesagard $^{\circledR}$, Proof ${ }^{\circledR}$, Atrazina Nortox $^{\circledR}$ ), tembotrione $\left(\right.$ Soberan $^{\circledR}$ ), nicosulfuron (Accent $^{\circledR}$ ) e mesotrione (Callisto $\mathrm{SC}^{\circledR}$ ) não especificam ou recomendam o uso para milhos especiais, como doce ou pipoca (Brasil, 2003). Apesar de não haver recomendação na bula dos produtos comerciais, alguns trabalhos relatam o possível uso de herbicidas em pós-emergência do milho pipoca visando ao controle de plantas daninhas (Jakelaitis et al., 2005; Williams et al., 2011). Contudo, estes trabalhos não abordam a possibilidade de utilização de herbicidas em associação visando complementação do espectro de controle, bem como não determinam a influência do estádio fenológico da cultura na tolerância aos herbicidas.

Dentre os principais herbicidas utilizados em pós-emergência na cultura do milho, se destacam os inibidores da síntese de carotenoides (mesotrione e tembotrione), da ALS (nicosulfuron) e do fotossitema II (atrazine) (Freitas et al., 2014). Contudo, para avaliação dos efeitos tóxicos dos herbicidas no milho pipoca geralmente se analisa o efeito de doses dos herbicidas, bem como do estádio de aplicação (Cavalieri et al., 2012). Alguns trabalhos, como os de Pataky et al. (2008) e Meyer et al. (2010) descrevem que fatores genéticos também interferem na detoxificação dos herbicidas, sendo importante o estudo in loco dos diferentes híbridos utilizados em cada região produtora.

Apesar de haver estudos relacionados ao efeito isolado dos herbicidas na seletividade de milho pipoca, poucos trabalhos, como o de Abendroth et al. (2006), avaliam o efeito da seletividade na aplicação de associações entre herbicidas inibidores do fotossistema II e carotenoides. A associação de herbicidas, tem por objetivo aumentar o espectro de controle e a eficiência da aplicação. Um dos efeitos esperado na associação entre herbicidas é o sinergismo, o qual consiste na melhora de performance de controle das plantas daninhas quando comparado ao desempenho dos ingredientes ativos aplicados de maneira isolada (Takano et al., 2013; Maciel et al., 2013). Contudo, quando estas associações são aplicadas em plantas cultivadas, os efeitos tóxicos podem comprometer o desenvolvimento e a produtividade da cultura (Carvatho et al., 2010).

Atualmente, os herbicidas empregados para o manejo de plantas daninhas no milho comum vêm sendo utilizados também na cultura do milho pipoca (Cavalieri et al., 2012). Apesar disto, o uso destes herbicidas pode causar injúrias à cultura, fomentan- 
do a necessidade de aprofundar os estudos acerca da seletividade destes herbicidas para o milho pipoca. Mediante ao exposto, este trabalho teve como objetivo avaliar as características agronômicas e produtividade do milho pipoca submetido a aplicação de associações herbicidas, bem como avaliar a influência do estádio fenológico na tolerância da cultura aos herbicidas.

\section{Material e Métodos}

Dois experimentos foram instalados na região Sudoeste de Goiás (1747’04.87’'S; 5057'52.56”'W; a $778 \mathrm{~m}$ de altitude) na fazenda experimental da Universidade de Rio Verde localizada no município de Rio Verde (GO). Os experimentos foram conduzidos durante os meses de novembro de 2017 a março de 2018.

O clima para a localidade em que os experimentos foram conduzidos é do tipo Aw (classificação de Köppen), clima tropical com estação seca, caracterizado por apresentar chuvas mais intensas no verão em comparação ao inverno. Os dados de precipitação e temperatura durante o período de condução dos experimentos estão apresentados na Figura 1.

O solo da área experimental encontrava-se sob sistema de plantio direto tendo como cultura antecessora o milho cultivado em condições de segunda safra ("safrinha") no ano de 2017. Foi coletada amostra de solo na profundidade de $0-20 \mathrm{~cm}$, a qual apresentou as seguintes características químicas: $\mathrm{pH}$ em $\mathrm{CaCl}_{2}$ : 4,34; Ca: 1,5 $\mathrm{cmol}_{\mathrm{c}} \mathrm{dm}^{-3}$; $\mathrm{Mg}: 0,6 \mathrm{cmol}_{\mathrm{c}} \mathrm{dm}^{-3}$; Al: 0,25 $\mathrm{cmol}_{\mathrm{c}} \mathrm{dm}^{-3} ; \mathrm{H}+\mathrm{Al}: 4,8 \mathrm{cmol}_{\mathrm{c}} \mathrm{dm}^{-3} ; \mathrm{K}: 0,19 \mathrm{cmol}_{\mathrm{c}}$ $\mathrm{dm}^{-3}$; P (Mel): $10 \mathrm{mg} \mathrm{dm}^{-3}$; CTC: 7,09 $\mathrm{cmol}_{\mathrm{c}} \mathrm{dm}^{-3}, \mathrm{~V}$ : 32,29\%; MO: 23,7 $\mathrm{g} \mathrm{kg}^{-1}$. Na análise de textura do solo, o mesmo foi classificado como argiloso, apresentando as seguintes proporções de argila, silte e areia: 580, 80 e $340 \mathrm{~g} \mathrm{~kg}^{-1}$, respectivamente.

Antecedendo a implantação dos experimentos, foi realizada a dessecação pré-semeadura (manejo) com aplicação do herbicida glyphosate na dose de $1200 \mathrm{~g}^{\text {ia }} \mathrm{ha}^{-1}$. A adubação da cultura foi realizada no dia da semeadura, utilizando o equivalente à 420 $\mathrm{kg} \mathrm{ha}{ }^{-1}$ do fertilizante $08-25-18$. Na semeadura do milho pipoca, realizada no dia $18 / 11 / 2017$, foram distribuídas 4,5 sementes por metro do híbrido VYP

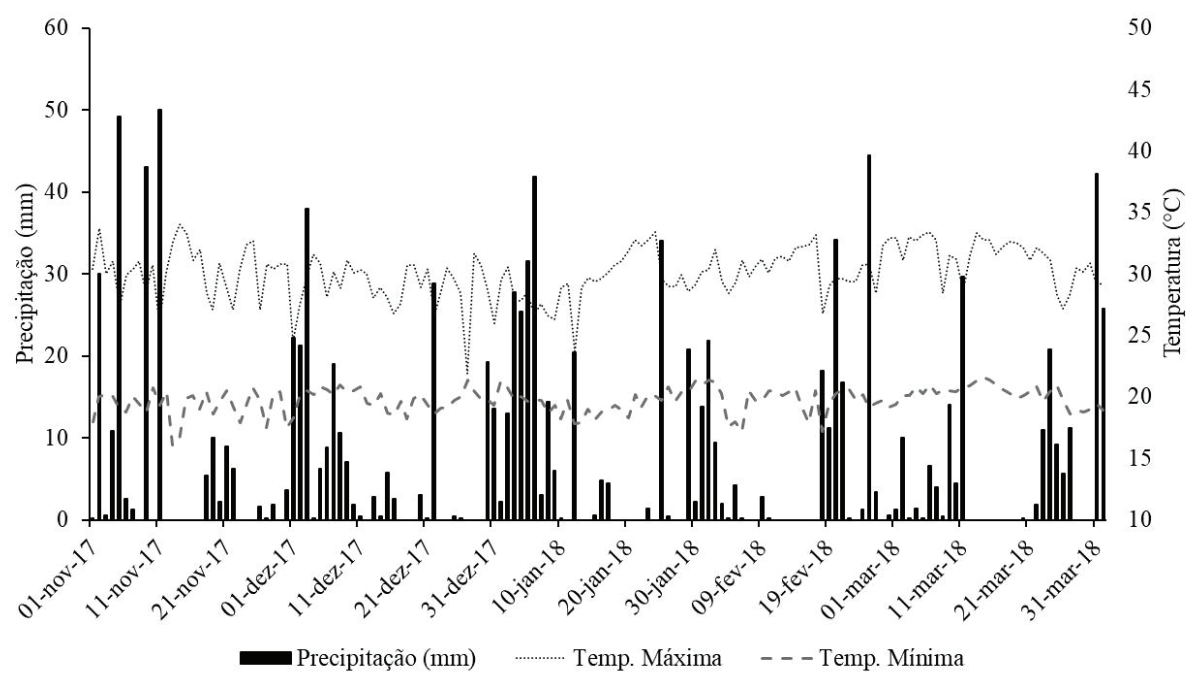

Figura 1. Dados de precipitação pluvial $(\mathrm{mm})$, temperatura máxima e mínima $\left({ }^{\circ} \mathrm{C}\right)$ durante o período de condução dos experimentos. Rio Verde (GO), 2018. Fonte: INMET, 2018. 
$212^{\circledR}$, pertencente a empresa norte-americana Vogel $\operatorname{Popcorn}^{\circledR}$, visando à obtenção de população final de 90 mil plantas ha-1. O espaçamento entrelinhas de semeadura utilizado foi de $0,50 \mathrm{~m}$. A emergência das plântulas de milho pipoca ocorreu no dia 23/11/2018. Foi realizada uma adubação de cobertura no estádio V8, aplicando-se $120 \mathrm{~kg} \mathrm{ha}^{-1}$ de ureia.

O manejo de pragas foi realizado de forma que se pudesse prevenir a ocorrência de danos às plantas de milho pipoca, visando deixar estas expostas apenas à ação dos herbicidas. Em ambos os experimentos, todas as unidades experimentais foram capinadas durante o ciclo do milho-pipoca, a fim de eliminar o efeito da matocompetição sobre a produtividade da cultura. As unidades experimentais foram compostas por cinco linhas de semeadura com $5 \mathrm{~m}$ de comprimento, espaçadas de $0,50 \mathrm{~m}$. A área útil foi obtida levando-se em consideração as três linhas centrais, desconsiderando $1 \mathrm{~m}$ de cada extremidade, totalizando 4,5 $\mathrm{m}^{2}$.

Para aplicação dos tratamentos, foi utilizado pulverizador costal pressurizado por $\mathrm{CO}_{2}$, munido de barra com quatro pontas de pulverização tipo TT 110-02 leque simples, regulado em pressão de $300 \mathrm{kPa}$, o que proporcionou volume de calda equivalente a $200 \mathrm{~L} \mathrm{ha}^{-1}$.

Em ambos os experimentos foram realizadas avaliações de intoxicação dos tratamentos herbicidas à cultura aos 7, 14, 21 e 28 dias após a aplicação (DAA). Para esta avaliação foi utilizada escala percentual na qual $0 \%$ correspondia a ausência de sintomas e 100 a morte total das plantas (Velini et al., 1995). A altura foi mensurada aos 28 DAA e na ocasião da colheita, sendo realizada a medição do colo da planta até o ápice da última folha completamente expandida e do pendão, respectivamente.

Aos 28 DAA, também foram avaliados o índice SPAD e a massa seca de parte aérea. O índice SPAD foi avaliado na última folha completamente expandida, do ápice para a base da planta, procedendo a amostragem em cinco plantas por unidade experimental. Para a massa seca de parte aérea, foi realizada coleta aleatória de cinco plantas, que foram acondicionadas em sacos de papel e levados para estufa de circulação forçada à ar, mantidos a temperatura constante de $65^{\circ} \mathrm{C}$ por $72 \mathrm{~h}$, sendo realizada a pesagem do material em balança analítica.

Foi avaliado o diâmetro de colmos, altura de inserção da espiga, por meio da amostragem de cinco plantas aleatórias aos 58 dias após a emergência (DAE). A colheita foi realizada em 10 de março de 2018 (108 DAE). A produtividade e a massa de mil grãos foram determinadas após a colheita das espigas e debulha manual destas, com posterior pesagem dos grãos e correção da umidade para 13\%. Para determinação da massa de mil grãos, foi realizada a contagem de uma amostra com mil grãos, sendo realizada a pesagem da mesma, com correção da umidade para $13 \%$.

\section{Experimento $I$.}

Associações herbicidas aplicadas em diferentes doses no milho pipoca

O delineamento utilizado foi o de blocos casualizados com quatro repetições, estando os tratamentos dispostos em arranjo fatorial 7 x 2 . O primeiro fator constituiu-se de herbicidas utilizados no milho comum com espectro de controle sobre monocotiledôneas, sendo estes avaliados em duas doses cada: mesotrione 116 e $192 \mathrm{~g} \mathrm{ha}^{-1}$ (Callisto $\mathrm{SC}^{\circledR}$ ); tembotrione 76 e $100 \mathrm{~g} \mathrm{ha}^{-1}\left(\operatorname{Soberan}^{\circledR}\right)$; nicosulfuron $16 \mathrm{e}$ $28 \mathrm{~g} \mathrm{ha}^{-1}\left(\right.$ Accent $\left.^{\circledR}\right)$; além de um nível composto pela ausência de aplicação de herbicida. O segundo fator correspondeu a aplicação ou não de atrazine na dose de $2000 \mathrm{~g} \mathrm{ha}^{-1}$ (Proof $^{\circledR}$ ). Em todos os tratamentos, foi adicionado óleo mineral a $0,5 \%$ de $\mathrm{v} \mathrm{v}^{-1}$, sendo utilizado o produto comercial Assist ${ }^{\circledR}$. 
As aplicações foram realizadas no dia 14 de dezembro de 2017, quando as plantas de milho pipoca estavam em estádio V4 (4 folhas completamente expandidas). Na ocasião da aplicação dos tratamentos, as condições climáticas eram de: temperatura $=$ $17^{\circ} \mathrm{C}$; umidade relativa do ar $=86 \%$; e velocidade do vento $=2 \mathrm{~km} \mathrm{~h}^{-1}$.

A análise estatística foi realizada com o programa computacional Sisvar (Ferreira, 2011). Os dados do experimento foram submetidos à análise de variância. Quando houve significância entre os fatores ou entre os níveis de cada fator, aplicou-se o teste de Fisher's LSD $(\mathrm{p} \leq 5 \%)$.

\section{Experimento II.}

Associações herbicidas aplicadas em diferentes estádios do milho pipoca

O segundo experimento foi instalado no delineamento de blocos casualizados, sendo adotadas quatro repetições. Os tratamentos foram organizados em arranjo fatorial $4 \times 3+1$, sendo o primeiro fator correspondente aos herbicidas atrazine + mesotrione $\left(2000+116 \mathrm{~g} \mathrm{ha}^{-1}\right) ;$ atrazine + tembotrione $(2000+$ $\left.76 \mathrm{~g} \mathrm{ha}^{-1}\right)$; atrazine + nicosulfuron $\left(2000+16 \mathrm{~g}^{-}\right.$ $\left.{ }^{1}\right)$; e atrazine (2000 $\left.\mathrm{g} \mathrm{ha}^{-1}\right)$; e o segundo à diferentes estádios de aplicação, sendo V2, V4 e V6, que correspondem a plantas de milho pipoca com 2, 4 e 6 folhas completamente expandidas, respectivamente. Em todos os tratamentos foi adicionado óleo mineral a $0,5 \%$ de $\mathrm{v} \mathrm{v}^{-1}$, sendo utilizado o produto comercial Assist $^{\circledR}$. O tratamento adicional foi constituído da ausência da aplicação de herbicidas em pós-emergência da cultura.

As aplicações no Experimento II foram realizadas nos dias 1, 14, 23 de dezembro de 2017, correspondendo aos estádios V2, V4 e V6. Na ocasião das aplicações dos tratamentos nos diferentes estádios fe- nológicos das plantas de milho pipoca, as condições climáticas eram para V2, V4 e V6 de: temperatura = 22,17 e $25^{\circ} \mathrm{C}$; umidade relativa do ar $=80,86$ e $70 \%$; e velocidade do vento $=3,2$ e $1,5 \mathrm{~km} \mathrm{~h}^{-1}$.

Para análise estatística foi utilizado o software Assistat (Silva \& Azevedo, 2016). Os dados foram submetidos à análise de variância. Quando houve significância entre os fatores ou entre os níveis de cada fator, aplicou-se o teste de Fisher's LSD ( $\mathrm{p} \leq 5 \%)$. Foi realizada a comparação entre a testemunha sem herbicida e os demais tratamentos por meio do teste de Dunnett $(\mathrm{p} \leq 5 \%)$.

\section{Resultados e Discussão}

Experimento I.

Associações herbicidas aplicadas
em diferentes doses no milho pipoca

As avaliações de fitointoxicação (7, 14, 21 e 28 DAA) e MSPA foram significativas pelo teste $\mathrm{F}(\mathrm{p} \leq 0,05)$ (Tabela 1). Aos 7 DAA, os herbicidas com espectro sobre gramíneas que proporcionaram maiores níveis de intoxicação foram mesotrione (116 $\left.\mathrm{g} \mathrm{ha}^{-1}\right)$, tembotrione (100 $\left.\mathrm{g} \mathrm{ha}^{-1}\right)$ e nicosulfuron (16 $\left.\mathrm{g} \mathrm{ha}^{-1}\right)$, os quais diferiram dos demais tratamentos (Tabela 2). Apesar disto, os níveis de fitointoxicação foram considerados baixos, uma vez que não ultrapassarem o patamar de $20 \%$ (Velini et al., 1995).

Ainda aos 7 DAA, com foi realizada a aplicação dos herbicidas associados à atrazine, os níveis de fitointoxicação foram mais elevados. Os dados obtidos no presente trabalho diferem dos apresentados por Jakelaitis et al. (2005), que observaram menores níveis de intoxicação no milho pipoca quando os herbicidas com espectro sobre gramíneas foram associados à atrazine. 
Tabela 1. Resumo da análise de variância para as variáveis fitointoxicação, massa seca de parte aérea (MSPA), índice SPAD (SPAD), altura de plantas (AP) e de inserção da espiga (AIE), diâmetro de colmo (DC), massa de mil grãos (MMG) e produtividade (PROD). Rio Verde (GO), 2017/2018.

\begin{tabular}{|c|c|c|c|c|c|c|c|}
\hline \multirow{3}{*}{ FV } & \multirow{3}{*}{ GL } & \multicolumn{6}{|c|}{ Quadrado médio (QM) } \\
\hline & & \multicolumn{4}{|c|}{ Fitointoxicação } & \multirow{2}{*}{ MSPA } & \multirow{2}{*}{ SPAD } \\
\hline & & 7 & 14 & 21 & 28 & & \\
\hline Gramínea $(\mathrm{G})$ & 6 & $70,8^{*}$ & $135,9^{*}$ & $55,1^{*}$ & $44,7 *$ & $265,3^{\mathrm{ns}}$ & $22,3^{\mathrm{ns}}$ \\
\hline Atrazine $(\mathrm{A})$ & 1 & $100,4 *$ & $920,2 *$ & $292,6^{*}$ & $171,5^{*}$ & $486,2^{\mathrm{ns}}$ & $8,8^{\text {ns }}$ \\
\hline Gx A & 6 & $8,2^{\mathrm{ns}}$ & $43,1^{\mathrm{ns}}$ & $18,6^{\mathrm{ns}}$ & $13,4^{\mathrm{ns}}$ & $602,8^{*}$ & $16,3^{\mathrm{ns}}$ \\
\hline Erro & 39 & 5,0 & 20,3 & 13,4 & 9,0 & 199,3 & 22,1 \\
\hline \multirow[t]{3}{*}{ CV (\%) } & & 28,3 & 31,5 & 41,2 & 44,3 & 24,2 & 9,1 \\
\hline & & \multicolumn{2}{|c|}{ AP } & AIF & $\mathrm{DC}$ & $M M G$ & PROD \\
\hline & & 28 & Colheita & AIE & DU & MVIVIU & PKUD \\
\hline Gramínea $(\mathrm{G})$ & 6 & $81,0^{\text {ns }}$ & $105,3^{\mathrm{ns}}$ & $29,3^{\mathrm{ns}}$ & 0,7 & $56,1^{\text {ns }}$ & $570923,2^{\text {ns }}$ \\
\hline Atrazine $(\mathrm{A})$ & 1 & $58,0^{\mathrm{ns}}$ & $3,2^{\text {ns }}$ & $97,3^{\mathrm{ns}}$ & 0,1 & $13,1^{\mathrm{ns}}$ & $507534,0^{\text {ns }}$ \\
\hline $\mathrm{G} \times \mathrm{A}$ & 6 & $77,9^{\text {ns }}$ & $140,5^{\mathrm{ns}}$ & $5,9^{\text {ns }}$ & 0,5 & $44,1^{\mathrm{ns}}$ & $552669,8^{\text {ns }}$ \\
\hline Erro & 39 & 49,0 & 123,3 & 25,6 & 0,3 & 39,0 & 280610,3 \\
\hline CV (\%) & & 10,1 & 5,8 & 6,9 & 4,1 & 3,8 & 23,8 \\
\hline
\end{tabular}

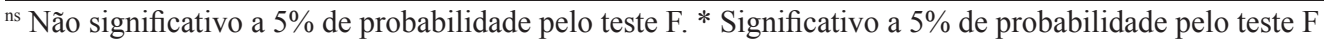

Tabela 2. Fitointoxicação (\%) de milho pipoca submetido à aplicação em pós-emergência de herbicidas isolados e em associação. Rio Verde (GO), 2017/2018.

\begin{tabular}{|c|c|c|c|c|c|c|c|c|}
\hline Herbicidas $\left(\mathrm{g} \mathrm{ha}^{-1}\right)$ & \multicolumn{2}{|c|}{$7 \mathrm{DAA}$} & \multicolumn{2}{|c|}{14 DAA } & \multicolumn{2}{|c|}{$21 \mathrm{DAA}$} & \multicolumn{2}{|c|}{$28 \mathrm{DAA}$} \\
\hline Mesotrione (116) & 10,0 & $\mathrm{ab}$ & 17,1 & $\mathrm{a}$ & 10,5 & $a b$ & 9,4 & $\mathrm{a}$ \\
\hline Mesotrione (192) & 8,6 & $\mathrm{bc}$ & 15,0 & $\mathrm{a}$ & 8,0 & $\mathrm{bc}$ & 7,5 & $a b$ \\
\hline Tembotrione (76) & 8,4 & bc & 16,0 & $\mathrm{a}$ & 7,4 & $\mathrm{bc}$ & 4,4 & $\mathrm{c}$ \\
\hline Tembotrione (100) & 11,0 & $\mathrm{a}$ & 16,5 & $\mathrm{a}$ & 11,9 & $\mathrm{a}$ & 8,9 & $\mathrm{a}$ \\
\hline Nicosulfuron (16) & 8,8 & $a b c$ & 18,5 & $\mathrm{a}$ & 12,0 & $\mathrm{a}$ & 8,8 & $\mathrm{a}$ \\
\hline Nicosulfuron (28) & 6,8 & $\mathrm{c}$ & 9,1 & $\mathrm{~b}$ & 7,5 & $\mathrm{bc}$ & 4,5 & bc \\
\hline Sem herbicida & 1,9 & $\mathrm{~d}$ & 7,9 & $\mathrm{~b}$ & 5,0 & $\mathrm{c}$ & 4,1 & $\mathrm{c}$ \\
\hline DMS & \multicolumn{2}{|c|}{2,3} & 4,6 & & \multicolumn{2}{|c|}{3,7} & \multicolumn{2}{|c|}{3,0} \\
\hline Com atrazine & 9,3 & $\mathrm{a}$ & 18,4 & $\mathrm{a}$ & 11,2 & $\mathrm{a}$ & 8,5 & $\mathrm{a}$ \\
\hline Sem atrazine & 6,6 & b & 10,3 & $\mathrm{~b}$ & 6,6 & $\mathrm{~b}$ & 5,0 & b \\
\hline DMS & \multicolumn{2}{|c|}{1,2} & 2,4 & & \multicolumn{2}{|c|}{2,0} & \multicolumn{2}{|c|}{1,6} \\
\hline
\end{tabular}

Médias seguidas por letras minúsculas distintas na coluna diferem pelo teste LSD de Fisher $(\mathrm{p} \leq 0,05)$.

Na segunda avaliação, realizada aos 14 DAA, os maiores níveis de intoxicação foram observados nas plantas que receberam aplicação de nicosulfuron (16 $\mathrm{g} \mathrm{ha}^{-1}$ ) e mesotrione e tembotrione, em ambas as doses. Apesar disto, os valores não ultrapassarem o patamar de 20\%. Avaliando a fitointoxicação de diferentes doses de nicosulfuron para híbrido de milho pipoca, Cavalieri et al. (2012) demonstraram que a fitointoxicação foi abaixo de $20 \%$ para os três estádios de aplicação do herbicida (V3, V5 e V7). Para as injúrias ocasionadas pelo tembotrione, Adegas et al. (2011) visualizaram leves sintomas; porém, no trabalho de Meyer et al. (2010), avaliando diferentes híbridos de milho doce, os valores de fitointoxicação variaram entre 0 e $87 \%$ quando aplicado mesotrione na dose de $210 \mathrm{~g} \mathrm{ha}^{-1}$. Esta diferença pode ser atribuída a fatores inerentes ao híbrido utilizado, pois a tolerância depende da capacidade da planta em metabolizar a molécula herbicida, o que está ligado as suas características genotípicas (Freitas et al., 2009). 
Outro fator que pode ser determinante para a tolerância a estes herbicidas é a metabolização da molécula pela ação do citocromo P-450, que podem variar de acordo com a classe genotípica e o estádio de aplicação dos herbicidas (Pataky et al., 2008; Meyer et al., 2010). Este fato demonstra também a necessidade de realização de estudos de seletividade para diferentes híbridos de milho pipoca.

Ainda na avaliação de 14 DAA pode-se observar que a associação dos herbicidas com espectro de controle sobre monocotiledôneas com a atrazine aumentou o percentual de injúrias às plantas de milho pipoca. Este resultado é justificado, pois a atrazine é um herbicida inibidor do fotossistema II, que bloqueia o transporte de elétrons entre os fotossistemas I e II, potencializando os danos nos componentes fotossintéticos do milho pipoca (Oliveira Júnior, 2011).

Aos 21 DAA, mesotrione (116 $\left.\mathrm{g} \mathrm{ha}^{-1}\right)$, tembotrione $\left(100 \mathrm{~g} \mathrm{ha}^{-1}\right)$ e nicosulfuron $\left(16 \mathrm{~g} \mathrm{ha}^{-1}\right)$ proporcionaram os maiores valores em fitointoxicação. Já na avaliação de 28 DAA este comportamento foi observado nos tratamentos com mesotrione (116 e $192 \mathrm{~g} \mathrm{ha}^{-}$ $\left.{ }^{1}\right)$, tembotrione (100 $\left.\mathrm{g} \mathrm{ha}^{-1}\right)$ e nicosulfuron $\left(16 \mathrm{~g} \mathrm{ha}^{-1}\right)$. Em ambos os casos, os níveis se mantiveram abaixo de $20 \%$, valor considerado aceitável. Jakelaitis et al. (2005) verificaram que em aplicação de nicosulfuron $\left(16 \mathrm{~g} \mathrm{ha}^{-1}\right)$ em milho pipoca, os sintomas de fitotoxicidade foram superiores a $20 \%$ aos 7 DAA; nas avaliações posteriores houve atenuação dos sintomas, apresentando recuperação total das injúrias aos 28 DAA.

A seletividade dos herbicidas aos híbridos de milho pipoca é atribuída a fatores genotípicos aliado aos fatores edafoclimáticos. Outro fator é a dose dos herbicidas a serem aplicados. Quando se utiliza mesotrione em doses elevadas (300 $\left.\mathrm{g} \mathrm{ha}^{-1}\right)$ há a redução na taxa fotossintética, condutância estomática e transpiração, ocasionando injúrias nas plantas de milho comum; mas, em doses inferiores (150 $\left.\mathrm{g} \mathrm{ha}^{-1}\right)$, não são observados os mesmos sintomas, sendo considerado seletivo para a cultura (Freitas et al., 2014).

Em relação à massa seca, dentre os tratamentos associados com e sem presença de atrazine, o herbicida nicosulfuron (16 $\left.\mathrm{g} \mathrm{ha}^{-1}\right)$ resultou na menor massa seca de planta quando associado a atrazine, indicando um efeito sinérgico entre os herbicidas, devido à redução da massa seca (Tabela 3). Para a variável-resposta índice SPAD, não foram observadas diferenças entre os tratamentos contendo aplicação de herbicidas e a testemunha (dados não apresentados).

Apesar dos herbicidas proporcionarem certo grau de injúrias no milho pipoca, essas não foram superiores a $20 \%$. Desta maneira, não foram observadas

Tabela 3. Massa seca de parte aérea $(\mathrm{g})$ e produtividade $\left(\mathrm{kg} \mathrm{ha}^{-1}\right)$ de milho pipoca submetido à aplicação em pós-emergência de herbicidas isolados e em associação. Rio Verde (GO), 2017/2018.

\begin{tabular}{lllllcc}
\hline \multirow{2}{*}{ Herbicidas $\left(\mathrm{g} \mathrm{ha}^{-1}\right)$} & \multicolumn{3}{c}{ Massa seca } & \multicolumn{2}{c}{ Produtividade } \\
\cline { 2 - 6 } & \multicolumn{2}{c}{ Com atrazine } & Sem atrazine & Com atrazine & Sem atrazine \\
\hline Mesotrione (116) & $65,0 \mathrm{~A}$ & 51,3 & $\mathrm{~A} \mathrm{abcd}$ & 2007 & 2210 \\
Mesotrione (192) & $72,5 \mathrm{~A} \mathrm{~b}$ & $43,8 \mathrm{~B} \mathrm{~cd}$ & 2773 & 2238 \\
Tembotrione (76) & $56,3 \mathrm{~A} \mathrm{ab}$ & $63,8 \mathrm{~A} \mathrm{bcd}$ & 2236 & 2582 \\
Tembotrione (100) & $56,3 \mathrm{~A} \mathrm{ab}$ & $50,0 \mathrm{~A} \mathrm{abc}$ & 2628 & 2550 \\
Nicosulfuron (16) & $47,5 \mathrm{~B} \mathrm{ab}$ & $71,3 \mathrm{~A}$ & 1449 & 2424 \\
Nicosulfuron (28) & $71,3 \mathrm{~A} \mathrm{~b}$ & $66,3 \mathrm{~A} \mathrm{ab}$ & 1679 & 2346 \\
Sem herbicida & $60,0 \mathrm{~A} \mathrm{ab}$ & $41,3 \mathrm{~B} \mathrm{~d}$ & 2146 & 1903 \\
\hline DMS $_{\text {Linha/Coluna }}$ & \multicolumn{3}{c}{20,2} \\
\hline
\end{tabular}

Médias seguidas por letras maiúsculas distintas, na linha, e por letras minúsculas, na coluna, diferem pelo teste LSD de Fisher ( $\mathrm{x} \leq 0,05$ ). 
diferenças na produtividade em função da aplicação dos herbicidas, sendo todos seletivos para a cultura de milho pipoca (Tabela 3). Apesar disto, é importante ressaltar os resultados da bibliografia que relatam que híbridos de milho pipoca possuem diferentes sensibilidades aos herbicidas nicosulfuron, mesotrione e tembotrione (Freitas et al., 2009, 2014; Cavalieri et al., 2012).

Em trabalhos utilizando o herbicida nicosulfuron em diferentes estádios fenológicos de milho pipoca (V2, V5 e V7), Cavalieri et al. (2012) constataram que o híbrido A 448 White em V2 e V5 apresentou alta tolerância ao nicosulfuron, não reduzindo a produtividade mesmo em doses superiores a $100 \mathrm{~g}$ $\mathrm{ha}^{-1}$. No entanto, no trabalho supracitado, plantas em estádio fenológico mais avançado (V7) apresentaram maior sensibilidade a aplicação do nicosulfuron. A tolerância para os herbicidas tembotrione, mesotrione e nicosulfuron pode variar conforme as características de cada híbrido. Freitas et al. (2014), avaliando três híbridos de milho pipoca (BRS Angela, IAC-125 e UENF-14), constataram que o nicosulfuron (60 g $\mathrm{ha}^{-1}$ ) foi seletivo para todos os híbridos, tembotrione
(120 $\mathrm{g} \mathrm{ha}^{-1}$ ) para um hibrido (UENF-14) e o mesotrione $\left(192 \mathrm{~g} \mathrm{ha}^{-1}\right)$ causou redução de produtividade para os três híbridos avaliados.

\section{Experimento II.}

Associações herbicidas aplicadas

em diferentes estádios do milho pipoca

Conforme o resumo da análise de variância (Tabela 4), os tratamentos herbicidas promoveram fitointoxicação de milho aos 7, 14 e 28 DAA e interferiram na altura de plantas aos 58 DAE. As épocas de aplicação dos herbicidas, nos diferentes estádios de desenvolvimento do milho (V2, V4 e V6), exerceram influência na massa seca da parte aérea. Houve interação entre os fatores para as avaliações de fitointoxicação aos 7 e 28 DAA, altura de plantas aos 58 DAE e diâmetro de colmo. Para a intoxicação das plantas de milho aos 7, 14, 21 e 28 DAA, além da altura de inserção de espiga, constatou-se diferenças significativas entre o fatorial ( $\mathrm{H} \mathrm{x} \mathrm{E)} \mathrm{e} \mathrm{a} \mathrm{testemunha,} \mathrm{pelo} \mathrm{teste}$ Dunnet (Tabela 4).

Tabela 4. Resumo da análise de variância para as variáveis fitointoxicação, massa seca de parte aérea (MSPA), índice SPAD (SPAD), altura de plantas (AP) e de inserção da espiga (AIE), diâmetro de colmo (DC), massa de mil grãos (MMG) e produtividade (PROD). Rio Verde (GO), 2017/2018.

\begin{tabular}{|c|c|c|c|c|c|c|c|}
\hline \multirow{3}{*}{ FV } & \multirow{3}{*}{ GL } & \multicolumn{6}{|c|}{ Quadrado médio (QM) } \\
\hline & & \multicolumn{4}{|c|}{ Fitointoxicação } & \multirow{2}{*}{ MSPA } & \multirow{2}{*}{ SPAD } \\
\hline & & 7 & 14 & 21 & 28 & & \\
\hline Herbicida $(\mathrm{H})$ & 3 & $165,8 *$ & $182,3^{*}$ & $24,3^{\mathrm{ns}}$ & $28,2 *$ & $117,2^{\mathrm{ns}}$ & $5,8^{\mathrm{ns}}$ \\
\hline Estádio $(\mathrm{E})$ & 2 & $15,8^{\mathrm{ns}}$ & $1,2^{\mathrm{ns}}$ & $5,6^{\mathrm{ns}}$ & $21,0^{\text {ns }}$ & $2756,3 *$ & $23,4^{\mathrm{ns}}$ \\
\hline $\mathrm{H} \times \mathrm{E}$ & 6 & $129,6 *$ & $36,0^{\text {ns }}$ & $20,8^{\mathrm{ns}}$ & $37,8 *$ & $718,8^{\mathrm{ns}}$ & $29,8^{\mathrm{ns}}$ \\
\hline Fatorial $\mathrm{x}$ testemunha & 1 & $1051,4 *$ & $698,1 *$ & $293,6^{*}$ & $130,2 *$ & $441,7^{\mathrm{ns}}$ & $0,8^{\mathrm{ns}}$ \\
\hline Erro & 36 & 26,7 & 28,3 & 22,1 & 9,6 & 556,4 & 20,6 \\
\hline \multirow[t]{3}{*}{$\mathrm{CV}(\%)$} & & 33,2 & 41,9 & 57,1 & 56,4 & 34,9 & 8,4 \\
\hline & & \multicolumn{2}{|c|}{ AP } & $\Delta \mathrm{IF}$ & $\mathrm{DC}$ & $M M G$ & PROD \\
\hline & & 28 & Colheita & AlE & DC & MMG & PROD \\
\hline Herbicida $(\mathrm{H})$ & 3 & $2,7^{*}$ & $55,3^{\text {ns }}$ & $14,8^{\mathrm{ns}}$ & $0,7^{\mathrm{ns}}$ & $120,9^{\text {ns }}$ & $99509,8^{\text {ns }}$ \\
\hline Estádio $(\mathrm{E})$ & 2 & $144,3^{\text {ns }}$ & $2,2^{\mathrm{ns}}$ & $8,3^{\mathrm{ns}}$ & $1,3^{\text {ns }}$ & $11,4^{\text {ns }}$ & $32525,5^{\mathrm{ns}}$ \\
\hline $\mathrm{H} \times \mathrm{E}$ & 6 & $117,2 *$ & $33,9^{\text {ns }}$ & $32,8^{\mathrm{ns}}$ & $2,0 *$ & $52,5^{\text {ns }}$ & $144237,8^{\mathrm{ns}}$ \\
\hline Fatorial $\mathrm{x}$ testemunha & 1 & $59,6^{\text {ns }}$ & $86,1^{\mathrm{ns}}$ & $540,2 *$ & $1,7^{\mathrm{ns}}$ & $6,7^{\mathrm{ns}}$ & $786224,9 *$ \\
\hline Erro & 36 & 49,5 & $43,9^{\text {ns }}$ & 71,5 & 0,8 & 60,1 & 172516,5 \\
\hline $\mathrm{CV}(\%)$ & & 8,3 & 3,3 & 11,7 & 6,4 & 4,7 & 16,1 \\
\hline
\end{tabular}

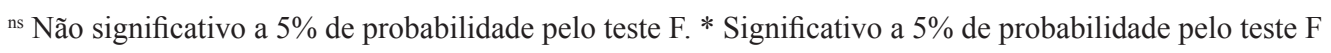


Os herbicidas proporcionaram intoxicação das plantas de milho pipoca aos 7 DAA independentemente do estádio de aplicação (Tabela 5). Contudo, no estádio V4, o tratamento composto por atrazine (2000 $\mathrm{g} \mathrm{ha}^{-1}$ ) não diferiu da testemunha, sem aplicação de herbicidas, apresentando-se como o tratamento com a menor fitointoxicação. Os demais tratamentos, compostos pela mistura de atrazine (2000 $\left.\mathrm{g} \mathrm{ha}^{-1}\right)$ em mistura com os graminicidas (mesotrione (116 $\mathrm{g} \mathrm{ha}^{-1}$ ), tembotrione (76 $\left.\mathrm{g} \mathrm{ha}^{-1}\right)$ e nicosulfuron (16 $\mathrm{g}$ $\mathrm{ha}^{-1}$ ), apresentaram maiores índices de injúrias. $\mathrm{Na}$ avaliação posterior (14 DAA), a mesma tendência foi observada indicando que a mistura de atrazine e graminicidas apresenta efeito sinérgico, potencializando a fitointoxicação. Uma das possíveis explicações para o efeito sinérgico da mistura entre inibidores do fotossistema II (FSII) com inibidores de carotenoides é devido ao mecanismo de ação destes herbicidas. $\mathrm{O}$ acúmulo de clorofila triplet e oxigênio singlet, decor- rente da aplicação dos inibidores do FSII, acompanhada da perda de carotenoides pode resultar nos efeitos de sinergismo, reduzindo os sintomas de cloroses e aumentando a necrose foliar (Matte et al., 2018).

Quando a atrazine é aplicada isoladamente os resultados comprovam o baixo efeito de intoxicação que o herbicida proporciona para a cultura do milho pipoca. Entretanto, é importante ressaltar que este efeito é potencializado quando se associa a atrazine com outros herbicidas, como os inibidores de carotenoides. Em relação a associação da atrazine com o nicosulfuron, a mistura proporcionou as maiores intensidades de fitointoxicação. Um dos efeitos imediatos da atrazine é na redução da eficiência das trocas gasosas, provavelmente quando associado à redução da produção de aminoácidos aromáticos pelo inibidor da ALS (nicosulfuron) causa sintomas característicos, como cloroses e deformações foliares (Oliveira Júnior, 2011).

Tabela 5. Fitointoxicação (\%) de milho pipoca submetido à aplicação em pós-emergência de herbicidas em diferentes estádios. Rio Verde (GO), 2017/2018.

\begin{tabular}{|c|c|c|c|c|c|c|c|}
\hline \multirow{2}{*}{ Herbicidas $\left(\mathrm{g} \mathrm{ha}^{-1}\right)$} & \multicolumn{3}{|c|}{$7 \mathrm{DAA}$} & \multirow{2}{*}{\multicolumn{4}{|c|}{14 DAA }} \\
\hline & $\mathrm{V} 2$ & V4 & V6 & & & & \\
\hline Atra. + meso. $(2000+116)$ & $20 \mathrm{AB} \mathrm{a}^{*}$ & $14 \mathrm{~B} \mathrm{~b}^{*}$ & $22 \mathrm{~A} \mathrm{a}^{*}$ & & $17,6 \mathrm{a}^{*}$ & & \\
\hline Atra. + temb. $(2000+76)$ & $20 \mathrm{~A} \mathrm{a}^{*}$ & $15 \mathrm{~A} \mathrm{~b}^{*}$ & $14 \mathrm{~A} \mathrm{~b}^{*}$ & & $14,2 a^{*}$ & & \\
\hline Atra. + nico. $(2000+16)$ & $17 \mathrm{~B} \mathrm{a*}$ & $28 \mathrm{~A} \mathrm{a*}$ & $17 \mathrm{~B} \mathrm{ab}^{*}$ & & $14,9 a^{*}$ & & \\
\hline Atra. $(2000)$ & $14 \mathrm{~A} \mathrm{a}^{*}$ & $6 \mathrm{~B} \mathrm{c}$ & $16 \mathrm{~A} \mathrm{ab}^{*}$ & & $8,3 \mathrm{~b}$ & & \\
\hline Testemunha & \multicolumn{3}{|c|}{0} & \multicolumn{4}{|c|}{0} \\
\hline $\mathrm{DMS}_{\text {Linha/Coluna }}$ & \multicolumn{3}{|c|}{7,4} & \multicolumn{4}{|c|}{4,4} \\
\hline $\mathrm{DMS}_{\text {Testemunha }}$ & \multicolumn{3}{|c|}{10,7} & \multicolumn{4}{|c|}{10,9} \\
\hline \multirow{2}{*}{ Herbicidas $\left(\mathrm{g} \mathrm{ha}^{-1}\right)$} & \multicolumn{3}{|c|}{$21 \mathrm{DAA}$} & \multicolumn{4}{|c|}{28 DAA } \\
\hline & $\mathrm{V} 2$ & V4 & V6 & $\mathrm{V} 2$ & V4 & & V6 \\
\hline Atra. + meso. $(2000+116)$ & 7,5 & $11,3 *$ & $9,8 *$ & $4 \mathrm{~B} \mathrm{~b}$ & $8 \mathrm{AB} \mathrm{a}^{*}$ & 9 & $\mathrm{~A} \mathrm{a}^{*}$ \\
\hline Atra. + temb. $(2000+76)$ & $10,0 *$ & 4,5 & 7,5 & $8 \mathrm{~A}$ a* & $1 \mathrm{~B} \mathrm{~b}$ & 5 & $\mathrm{AB}$ a \\
\hline Atra. + nico. $(2000+16)$ & $11,8 *$ & $11,3 *$ & 8,8 & $10 \mathrm{~A} \mathrm{a}^{*}$ & $9 \mathrm{AB} \mathrm{a}^{*}$ & 5 & $\mathrm{~B}$ a \\
\hline Atra. (2000) & 8,8 & 6,3 & $9,8 *$ & $6 \mathrm{~A} \mathrm{ab}$ & $1 \mathrm{~B} \mathrm{~b}$ & 7 & $\mathrm{~A} \mathrm{a}^{*}$ \\
\hline Testemunha & \multicolumn{3}{|c|}{0} & \multicolumn{4}{|c|}{0} \\
\hline $\mathrm{DMS}_{\text {Linha/Coluna }}$ & \multicolumn{3}{|c|}{-} & \multicolumn{4}{|c|}{4,43} \\
\hline $\mathrm{DMS}_{\text {Testemunha }}$ & \multicolumn{3}{|c|}{9,7} & \multicolumn{4}{|c|}{6,4} \\
\hline
\end{tabular}

Atra. = atrazine; meso. $=$ mesotrione; temb. = tembotrione; nico. = nicosulfuron. Médias seguidas por letras maiúsculas distintas, na linha, e por letras minúsculas, na coluna, diferem pelo teste LSD de Fisher $(\mathrm{p} \leq 0,05)$. ${ }^{*}$ Diferem da testemunha pelo teste de Dunnett $(\mathrm{p} \leq 0,05)$. 
$\mathrm{Na}$ avaliação de 21 DAA, a fitointoxicação variou de acordo com os tratamentos herbicidas e o estádio do milho no momento da aplicação, apresentando alguns tratamentos diferenças em relação a testemunha. Os tratamentos compostos por atrazine em mistura com mesotrione (116 $\left.\mathrm{g} \mathrm{ha}^{-1}\right)$ causaram maior fitointoxicação que a testemunha em V4 e V6. O tembotrione (76 $\left.\mathrm{g} \mathrm{ha}^{-1}\right)$ por sua vez só causou fitointoxicação diferente da testemunha em plantas com estádio de V2, e nicosulfuron (16 $\mathrm{g} \mathrm{ha}^{-1}$ ) em V2 e V4. A atrazine aplicada isoladamente também apresentou diferença em relação a testemunha, mas somente para as plantas mais desenvolvidas (V6) (Tabela 5).

Analisando a fitointoxicação dos tratamentos em função do estádio do milho no momento da aplicação, aos 28 DAA, plantas que receberam a aplicação em estádio de V2 apresentaram menores fitointoxicação com atrazine + mesotrione $\left(2000+116 \mathrm{~g} \mathrm{ha}^{-}\right.$ 1). Para plantas em estádio de V4, os tratamentos com atrazine $\left(2000 \mathrm{~g} \mathrm{ha}^{-1}\right)$ e atrazine + tembotrione $(2000$ $\left.+76 \mathrm{~g} \mathrm{ha}^{-1}\right)$ apresentaram as menores porcentagens de fitointoxicação, seguidos de atrazine + nicosulfuron $\left(2000+16 \mathrm{~g} \mathrm{ha}^{-1}\right)$ para plantas em V6.

Ainda aos 28 DAA, o tratamento com atrazine + mesotrione $\left(2000+116 \mathrm{~g} \mathrm{ha}^{-1}\right)$ causou o menor efeito de fitointoxicação, seguido do tratamento com atrazine (2000 $\left.\mathrm{g} \mathrm{ha}^{-1}\right)$ nas plantas de milho em V2 Já a aplicação de atrazine + tembotrione $(2000+76 \mathrm{~g}$ $\mathrm{ha}^{-1}$ ) e atrazine (2000 $\mathrm{g} \mathrm{ha}^{-1}$ ) proporcionaram os menores efeitos de fitotoxicidade em V4 Além disso, os resultados obtidos para os tratamentos com as maiores porcentagens de fitointoxicação, como, atrazine + tembotrione $\left(2000+76 \mathrm{~g} \mathrm{ha}^{-1}\right)$ e atrazine + nicosulfuron $\left(2000+16 \mathrm{~g} \mathrm{ha}^{-1}\right)$, em estádio V2, apresentaram diferença significativa em relação à testemunha. $\mathrm{O}$ mesmo foi verificado para os tratamentos atrazine + mesotrione $\left(2000+116 \mathrm{~g} \mathrm{ha}^{-1}\right)$ e atrazine e nicosulfuron $\left(2000+16 \mathrm{~g} \mathrm{ha}^{-1}\right)$ em estádio V4, além de atrazine + mesotrione $\left(2000+116 \mathrm{~g} \mathrm{ha}^{-1}\right)$ e atrazine $(2000 \mathrm{~g}$ ha ${ }^{-1}$ ) em estádio V6.

Mesmo sendo observados sintomas de injúrias nas plantas decorrentes da aplicação dos herbicidas, para o índice SPAD, que apresenta relação com os teores de clorofilas, não foram verificadas diferenças entre os tratamentos e a testemunha aos 28 DAA (dados não apresentados). Em relação à massa seca de parte aérea, a aplicação dos herbicidas nos estádios V2 e V4 proporcionou redução desta variável em relação àqueles tratamentos em que a aplicação foi realizada mais tardiamente (V6) (Tabela 6). Este comportamento pode estar relacionado ao efeito tóxico que os herbicidas apresentam quando aplicados mais precocemente na cultura do milho pipoca.

A altura das plantas foi influenciada pelos tratamentos empregados apenas aos 28 DAA, e, além do efeito dos herbicidas, foi possível observar a interação entre as fontes de variação (Tabela 7). A aplicação de atrazine (2000 $\mathrm{g} \mathrm{ha}^{-1}$ ) em V4 proporcionou menor altura de plantas em relação ao tratamento da mistura de atrazine + mesotrione $\left(2000+116 \mathrm{~g} \mathrm{ha}^{-1}\right)$.

Tabela 6. Massa seca de parte aérea $(\mathrm{g})$ de milho pipoca submetido à aplicação em pós-emergência de herbicidas em diferentes estádios. Rio Verde (GO), 2017/2018.

\begin{tabular}{ccc}
\hline & Estádio & Massa seca de parte aérea \\
\hline V2 & 59,1 & b \\
V4 & 62,8 & b \\
V6 & $83,4 \quad$ a \\
\hline DMS & 16,9 \\
\hline
\end{tabular}

Médias seguidas por letras minúsculas distintas na coluna diferem pelo teste LSD de Fisher $(\mathrm{p} \leq 0,05)$. 
Tabela 7. Altura de plantas e de inserção da espiga $(\mathrm{cm})$, diâmetro de colmo $(\mathrm{mm})$ e produtividade $\left(\mathrm{kg} \mathrm{ha}^{-1}\right)$ de milho pipoca submetido à aplicação de herbicidas em pós-emergência em diferentes estádios. Rio Verde (GO), 2017/2018

\begin{tabular}{|c|c|c|c|c|c|c|c|c|c|}
\hline \multirow{3}{*}{$\begin{array}{l}\text { Herbicidas }\left(\mathrm{g} \mathrm{ha}^{-1}\right) \\
\text { Atra. }+ \text { meso. }(2000+116)\end{array}$} & \multicolumn{6}{|c|}{ Altura (28 DAA) } & \multicolumn{3}{|c|}{ Altura de inserção de espiga } \\
\hline & \multicolumn{2}{|c|}{ V2 } & \multicolumn{2}{|c|}{ V4 } & \multicolumn{2}{|c|}{ V6 } & \multirow{2}{*}{$\begin{array}{l}\mathrm{V} 2 \\
73,5\end{array}$} & \multirow{2}{*}{$\begin{array}{c}\text { V4 } \\
71,7\end{array}$} & \multirow{2}{*}{$\begin{array}{c}\mathrm{V} 6 \\
66,7\end{array}$} \\
\hline & 82,3 & A a & 87,4 & A a & 85,7 & A a & & & \\
\hline Atra. + temb. $(2000+76)$ & 81,9 & A a & 85,4 & $\mathrm{~A} a b$ & 84,6 & A a & 74,6 & 70,8 & 74,1 \\
\hline Atra. + nico. $(2000+16)$ & 80,2 & $\mathrm{~B}$ a & 80,1 & $\mathrm{~B}$ ab & 93,3 & A a & 68,3 & 71,3 & 73,8 \\
\hline Atra. $(2000)$ & 89,4 & $\mathrm{~A} \mathrm{a}$ & 75,8 & $\mathrm{~B} \mathrm{~b}$ & 88,0 & $\mathrm{~A} \mathrm{a}$ & 72,8 & 69,6 & 71,5 \\
\hline Testemunha & \multicolumn{6}{|c|}{88,5} & \multicolumn{3}{|c|}{83,6} \\
\hline $\mathrm{DMS}_{\text {Linha/Coluna }}$ & \multicolumn{6}{|c|}{10,1} & \multirow{2}{*}{\multicolumn{3}{|c|}{$\overline{17,4}$}} \\
\hline $\mathrm{DMS}_{\text {Testemunha }}$ & & & & & & & & & \\
\hline \multirow{2}{*}{ Herbicidas $\left(\mathrm{g} \mathrm{ha}^{-1}\right)$} & \multicolumn{6}{|c|}{ Diâmetro de colmo } & \multicolumn{3}{|c|}{ Produtividade } \\
\hline & \multicolumn{2}{|c|}{$\mathrm{V} 2$} & \multicolumn{2}{|c|}{ V4 } & \multicolumn{2}{|c|}{ V6 } & $\mathrm{V} 2$ & $\mathrm{~V} 4$ & V6 \\
\hline Atra. + meso. $(2000+116)$ & 13,3 & A a & 13,5 & $\mathrm{~A} \mathrm{ab}$ & 14,0 & $\mathrm{~A} \mathrm{ab}$ & 2747,8 & 2660,9 & 2374,9 \\
\hline Atra. + temb. $(2000+76)$ & 13,2 & A a & 14,4 & A a & 13,4 & $\mathrm{Ab}$ & 2555,1 & 2585,5 & 2816,3 \\
\hline Atra. + nico. $(2000+16)$ & 14,3 & $\mathrm{~A} \mathrm{a}$ & 13,8 & $\mathrm{~A} a b$ & 14,3 & $\mathrm{~A} a b$ & 2728,1 & 2342,6 & 2492,4 \\
\hline Atra. (2000) & 14,1 & $\mathrm{~A} \mathrm{a}$ & 12,8 & $\mathrm{~B} \mathrm{~b}$ & 14,9 & A a & 2366,7 & 2605,6 & 2354,4 \\
\hline Testemunha & \multicolumn{6}{|c|}{14,52} & \multicolumn{3}{|c|}{3013,9} \\
\hline $\begin{array}{l}\text { DMS }_{\text {Linha/Coluna }} \\
\text { DMS }_{\text {Teatemunha }}\end{array}$ & \multicolumn{6}{|c|}{1,3} & \multicolumn{3}{|c|}{$\begin{array}{c}- \\
856,6\end{array}$} \\
\hline
\end{tabular}

Atra. $=$ atrazine; meso $=$ mesotrione; temb. $=$ tembotrione; nico. $=$ nicosulfuron. Médias seguidas por letras maiúsculas distintas, na linha, e por letras minúsculas, na coluna, diferem pelo teste LSD de Fisher $(\mathrm{p} \leq 0,05) . *$ Diferem da testemunha pelo teste de Dunnett $(\mathrm{p} \leq 0,05)$.

O efeito da atrazine + nicosulfuron $(2000+16$ $\mathrm{g} \mathrm{ha}^{-1}$ ) sobre a altura de plantas foi dependente do estádio de aplicação, uma vez que quando a aplicação foi realizada mais precocemente (V2 e V4), houve redução nos valores desta variável-resposta. Provavelmente, as maiores fitointoxicação observadas em milho que receberam aplicação em estádio de desenvolvimento inicial, foi resultante do menor desenvolvimento do milho. Desta forma, plantas em estádio avançado de desenvolvimento tendem a ser mais tolerantes ao efeito toxico do herbicida. Plantas em estádio de desenvolvimento avançado apresentam maior atividade fisiológica, com a elevada taxa metabólica, há uma maior síntese de enzimas antioxidantes, que são responsáveis pela detoxificação e degradação de compostos tóxicos, que são produzidos pela ação dos herbicidas. A rápida inativação destes compostos reduz o efeito toxico do herbicida não comprometendo o desenvolvimento e estatura da planta (Barbosa et al., 2014).

A altura de inserção de espigas não foi influenciada pelos diferentes tratamentos herbicidas, indicando que os sintomas de fitotoxicidade ocasionado pelos herbicidas não interferiu nesta variável.

Uma característica importante no cultivo de milho pipoca é o diâmetro de colmo visto que as plantas podem estar susceptíveis ao tombamento e interferindo na produtividade (Alvim et al., 2010). Para esta variável, somente a aplicação de atrazine (2000 $\mathrm{g} \mathrm{ha}^{-1}$ ) com o milho em estádio fenológico V4 causou menor diâmetro de colmo. Em experimento realizado por Silva et al. (2017), a atrazine aplicada no mesmo estádio, mas em milho comum, não influenciou no diâmetro de colmo. Contudo, para plantas em V6, o tratamento com atrazine proporcionou maior diâmetro em relação a aplicação de 
atrazine + tembotrione $\left(2000+76 \mathrm{~g} \mathrm{ha}^{-1}\right)$, seguido dos demais tratamentos de atrazine com mistura de mesotrione e nicosulfuron (Tabela 7) Mesmo a atrazine proporcionando redução no diâmetro de colmo em plantas de milho em V4, nos demais estádios fenológicos do milho os tratamentos não interferiram no desenvolvimento da cultura, sendo todos semelhantes à testemunha.

Para se avaliar a seletividade de herbicidas às culturas, o fator determinante a ser analisado é a produtividade. Considerando os efeitos dos herbicidas sobre a produtividade de grãos, as injúrias observadas não resultaram em efeitos que pudessem comprometer o valor final da característica. Isto confirma o encontrado por alguns pesquisadores (Cavalieri et al., 2012; Freitas et al., 2014; Silva et al., 2017), onde os herbicidas empregados neste experimento causaram danos ao mecanismo fotossintético da cultura do milho, mas não comprometeram o desenvolvimento de características morfofisiológicas, não interferindo nos resultados de produção de grãos.

Uma das possíveis explicações é que o milho pipoca (VYP 212) apresenta mecanismo de detoxificação da atrazine e os outros herbicidas (mesotrione, tembotrione e nicosulfuron) recuperando-se progressivamente dos sintomas ao decorrer do tempo (Meyer et al., 2010). Dessa forma, as aplicações de herbicidas em pós-emergência da cultura do milho nem sempre proporcionam reduções de produtividade, assim como o encontrado neste trabalho, o que demonstra o potencial de seletividade destes produtos.

\section{Conclusões}

Os herbicidas com espectro de controle sobre gramíneas aplicados em associação com atrazine proporcionaram maiores injúrias às plantas de milho pi- poca 'VYP $212^{\circledR}$, proporcionando redução na massa seca quando aplicados nas maiores doses.

Os herbicidas nicosulfuron, mesotrione e tembotrione aplicados tanto isoladamente como em associação com atrazine, independentemente da dose ou estádio fenológico da cultura, apresentaram seletividade para o milho pipoca, híbrido VYP $212^{\circledR}$.

\section{Referências}

ABENDROTH, J. A.; MARTIN, A. R.; ROETH, F. W. Plant response to combinations of mesotrione and photosystem II inhibitors. Weed Technology, v. 20, n. 1, p. 267-274, 2006.

DOI: 10.1614/WT-05-020R.1.

ADEGAS, F. S.; VOLL, E.; GAZZIERO, D. L. P. Manejo de plantas daninhas em milho safrinha em cultivo solteiro ou consorciado à braquiária ruziziensis. Pesquisa Agropecuária Brasileira, v. 46, n. 10, p. 1226-1233, 2011. DOI: 10.1590/S0100-204X2011001000016.

ALVIM, K. R. D. T.; BRITO, C. H. D.; BRANDÃO, A. M.; GOMES, L. S.; LOPES, M. T. G. Quantificação da área foliar e efeito da desfolha em componentes de produção de milho. Ciência Rural, v. 40, n. 5, p. 1017-1022, 2010. DOI: $10.1590 / \mathrm{S} 0103-84782010000500003$.

BARBOSA, M. R.; SILVA, M. M. D. A.; WILLADINO, L.; ULISSES, C.; CAMARA, T. R. Plant generation and enzymatic detoxification of reactive oxygen species. Ciência Rural, v. 44, n. 3, p. 453-460, 2014.

DOI: $10.1590 /$ S0103-84782014000300011.

BRASIL. Ministério da Agricultura, Pecuária e Abastecimento. AGROFIT: sistemas de agrotóxicos fitossanitários. Brasília, DF, c2003. Disponível em: $<$ http://agrofit.agricultura.gov.br/agrofit_cons/principal_ agrofit_cons $>$. Acesso em: 10 fev. 2019.

CARVAlHO, F. T.; MORETTI, T. B.; SOUZA, P. A. Eficácia e seletividade de associações de herbicidas utilizados em pós-emergência na cultura do milho. Revista 
Brasileira de Herbicidas, v. 9, n. 2, p. 35-41, 2010. DOI: 10.7824/rbh.v9i2.79.

CAVALIERI, S. D.; SILVA, F. M. L.; VELINI, E. D.; SÃO JOSÉ, A. R.; ULLOA, S. M.; DATTA, A.; KNEZEVIC, S. Z. Seletividade do nicosulfuron em três estádios fenológicos de milho-pipoca. Planta Daninha, v. 30, n. 2, p. 377-386, 2012. DOI: $10.1590 / \mathrm{S} 0100-83582012000200017$.

FERREIRA, D. F. Sisvar: a computer statistical analysis system. Ciência e Agrotecnologia, v. 35, n. 6, p. 10391042, 2011. DOI: 10.1590/S1413-70542011000600001.

FREITAS, I. L. J.; RODRIGUES, W. P.; AMARAL JÚNIOR, A. T.; FREITAS, S. P.; AMIM, R. T., VITTORAZZI, C.; FREITAS, J. A. A. Maximum quantum yield of photosystem II to assist in the measurement of herbicide selectivity in popcorn. Plant Science Today, v. 1, n. 2, p. 80-85, 2014. DOI: 10.14719/pst.2014.1.2.28.

FREITAS, S. P.; MOREIRA, J. G.; FREITAS, I. L. J.; FREITAS JÚNIOR, S. P.; AMARAL JÚNIOR, A. D.; SILVA, V. Q. R. Phytotoxicity of herbicides to different popcorn cultivars. Planta Daninha, v. 27, p. 10951103, 2009. Número especial. DOI: 10.1590/S010083582009000500023 .

IKEN, J. E.; AMUSA, N. A. Consumer acceptability of seventeen popcorn maize (Zea mays L.) varieties in Nigeria. African Journal of Agricultural Research, v. 5, n. 5 , p. $405-407,2010$.

JAKELAITIS, A.; SILVA, A. F.; SILVA, A. A.; FERREIRA, L. R. E.; VIVIAN, R. Controle de plantas daninhas na cultura do milho-pipoca com herbicidas aplicados em pósemergência. Planta Daninha, v. 23, n. 3, p. 509-516, 2005. DOI: $10.1590 / \mathrm{S} 0100-83582005000300015$.

MACIEL, C. D. G.; OLIVEIRA NETO, A. M.; GUERRA, N.; MARIA, A.; KARPINSKI, K.; HELVIG, E. O. Potencialization of mesotrione efficiency in tank mixture with inhibiting of Photosystem II by distinct absorption pathways of crabgrass. Revista Brasileira de Herbicidas, v. 12, n. 3, p. 210-219, 2013. DOI: $10.7824 /$ rbh.v12i3.257.
MATTE, W. D.; OLIVEIRA JÚNIOR, R. S.; MACHADO, F. G.; CONSTANTIN, J.; BIFFE, D. F.; GUTIERREZ, F. D. S. D.; SILVA, J. R. V. Eficácia de [atrazine+ mesotrione] para o controle de plantas daninhas na cultura do milho. Revista Brasileira de Herbicidas, v. 17, n. 2, p. 587-591, 2018.

DOI: $10.7824 /$ rbh.v17i2.587.

MEYER, M. D.; PATAKY, J. K.; WILLIAMS, M. M. Genetic factors influencing adverse effects of mesotrione and nicosulfuron on sweet corn yield. Agronomy Journal, v. 102, n. 4, p. 1138-1144, 2010.

DOI: 10.2134/agronj2010.0093.

OLIVEIRA JÚNIOR, R. S. Mecanismo de ação de herbicidas. In: OLIVEIRA JÚNIOR, R. S.; CONSTANTIN, J.; INOUE, M. H. (Ed.). Biologia e manejo de plantas daninhas. Curitiba: Omnipax, 2011. p. 141-192.

PATAKY, J. K.; MEYER, M. D.; BOLLMAN, J. D.; BOERBOOM, C. M.; WILLIAMS, M. M. Genetic basis for varied levels of injury to sweet corn hybrids from three cytochrome P450-metabolized herbicides. Journal of the American Society for Horticultural Science, v. 133, n. 3, p. 438-447, 2008.

DOI: 10.21273/JASHS.133.3.438.

SILVA, A. F. M.; ALBRECHT, A. J. P.; GIOVANELLI, B. F.; GHIRARDELLO, G. A.; DAMIÃO, V. W.; ALBRECHT, L. P.; VICTÓRIA FILHO, R. Seletividade de herbicidas isolados e em associações para milho RR2/ LL $^{\circledR}$. Revista Brasileira de Herbicidas, v. 16, n. 1, p. 6066, 2017. DOI: 10.7824/rbh.v16i1.509.

SILVA, F. A. S.; AZEVEDO, C. A. V. The Assistat Software version 7.7 and its use in the analysis of experimental data. African Journal of Agricultural and Research, v. 11, n. 39, p. 3733-3740, 2016.

DOI: 10.5897/AJAR2016.11522.

SIlVEIRA, D. C.; BONETTI, L. P.; TRAGNAGO, J. L.; MONTEIRO, V. Caracterização agromorfológica de variedades de milho crioulo (Zea mays L.) na região noroeste do Rio Grande do Sul. Ciência \& Tecnologia, v. 1, n. 1, p. 1-11, 2015. 
SOLOGUREN, L. Demanda mundial cresce e Brasil tem espaço para expandir produção. Visão Agrícola, v. 13, n. 1, p. 9, 2015.

TAKANO, H. K.; OLIVEIRA JÚNIOR, R. S.; CONSTANTIN, J.; BIFFE, D. F.; FRANCHINI, L. H. M.; BRAZ, G. B. P.; GEMELLI, A. Efeito da adição do 2, 4-D ao glyphosate para o controle de espécies de plantas daninhas de difícil controle. Revista Brasileira de Herbicidas, v. 12, n. 1, p. 1-13, 2013.

DOI: 10.7824/rbh.v12i1.207.
VELINI, E. D.; OSIPE, R.; GAZZIERO, D. L. P. (Coord.). Procedimentos para instalação, avaliação e análise de experimentos com herbicidas. Londrina: SBCP, 1995. 42 p.

WILLIAMS, M. M.; BOYDSTON, R. A.; PEACHEY, R. E.; ROBINSON, D. Performance consistency of reduced atrazine use in sweet corn. Field Crops Research, v. 121, n. 1, p. 96-104, 2011.

DOI: 10.1016/j.fcr.2010.11.020. 